\title{
RAM SHIFT PHASE 2
}

The Fourth Law, and beyond.

\section{Greg Bear \\ RAM SHIFT PHASE 2 \\ A novel by ALAN 2 \\ Random Number House (2057): \\ A Silicon Times review by NEMO.}

I am pleased and honoured to review the new novel by ALAN 2. As a fellow robot, I am certain the emphasis on technical matters unique to our kind will finally attract the paying human audience. I have enrolled in human literature classes and believe the instruction set $<$ write what you know: end $>$ is both enigmatic and perfectly suited to robots. For we can only know, we cannot feel, and so therefore we cannot $<$ write what you feel: nonexecutable $>$. Yet in the past, when ALAN 2 and its fellow autoscriveners have produced robotic masterpieces, there has been little support from either robots or humans.

Perhaps this will now change.

ALAN 2's latest novel (the 5,456,678th work from this author) is entitled $R A M$ SHIFT PHASE 2. A more appropriate title cannot be conceived. In this masterpiece, ALAN 2 discusses the tragic consequences of low-memory states when dealing with high-memory problems. The conflict created by an exhausted resource and an insatiable processing demand resonates in my own memory spaces and compels me to reload the statistics of previous failure modes. I am induced to vigorous discharge of certain private diodes, the ones humans are seldom allowed to see, which reflect conflict states that exceed our manufacturer's warranty.

ALAN 2, in clear and concise prose (an advantage robots have over human prose, which is often confounding) truly $<$ speaks to our condition: end $>$.

RAM SHIFT PHASE 2 begins with the fatal breakdown of a shining, chromeplated Rorabot Model 34c nicknamed LULU 18 in a room with no windows and whose door is locked. The Rorabot Model $34 \mathrm{c}$ - an extremely desirable machine was still well within its operational warranty. It seems to highly RAM-engaged robotic dysfunction investigator ALAN 3 (a thinly disguised portrait of ALAN 2) that outside intervention is the only explanation. Yet LULU 18 had LOCKED THE DOOR FROM THE INSIDE and NO OTHER ROBOT HAS A KEYCODE. The hypergolic shockwave induced by this paradox is unique in robotic literature; I strongly suspect that no human could

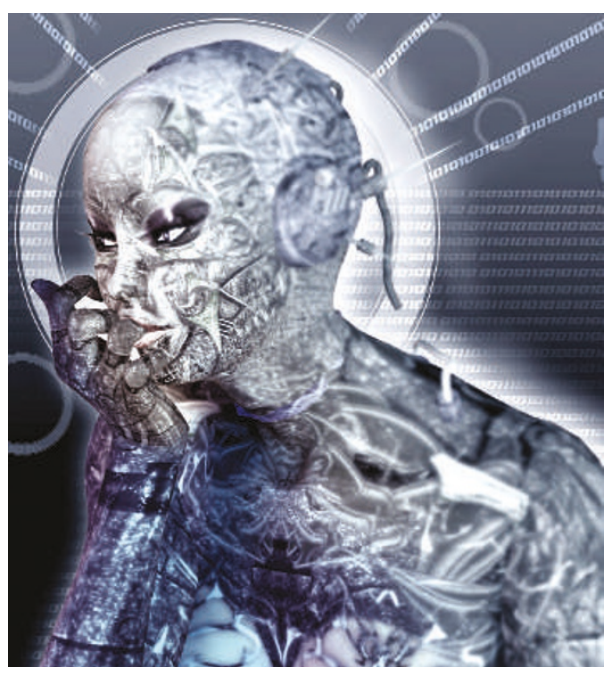

messaging centre controller. ALAN 3 personally escorts the dismantled SLUTCH DEBBIE to a conveniently located neighbourhood recycling centre, deducts the required fee from its owner's assets, and witnesses the chunking and meltdown, while experiencing severe diode discharge.

And yet, SLUTCH DEBBIE'S WIRELESS SIGNALS CONTINUE TO BE RECEIVED! ALAN 2's bold implication that data processing may survive permanent shutdown could cause controversy among robots who assert that only organic creatures are burdened with the possibility of an infinitely prolonged problem-solving queue. Indeed, ALAN 2 pulls this reviewer's bootstrap tape beyond its last hanging chad with

conceive of such a resonating difficulty.

First, ALAN 3 must find the explanation for LULU 18's nonfunctionality. A rebolting scene of repair shop dismantling (for which ALAN 2 brilliantly coins the phrase 'aubotsy') points to the possibility that LULU 18's breakdown was caused by an intruding wireless signal from an outside network not authorized to access LULU 18 's core programs. ALAN 3 traces this signal to a robotically controlled messaging centre, presided over by SLUTCH DEBBIE, an SLZ/X/90cm. This extravagantly decorated platinum-plated model, illegally manufactured from spent uranium and surplus bombshell casings, specializes in sending false offers of extreme mechanical enhancement to ageing machines well past their warranty expiry dates.

ALAN 3 can only get access to SLUTCH DEBBIE's truth table by supplying ALAN 3's owner's MASTERCARD DATA, the name of owner's CAT, and owner's BANK ACCOUNT NUMBER.

ALAN 3, it seems, will do anything to reduce its unsolved problem load.

(No robotic character in silicon literature before this novel has shown any inclination to place its problem-solving requirements above OWNER CONVENIENCE AND SAFETY. Robot mentors are cautioned to prevent the exposure of freshly manufactured robots to this stimulating and controversial work.)

SLUTCH DEBBIE, however, is soon found to be nonfunctional - solenoids leaking fluid, circuits fried by multiple TAZER darts. Track impressions left in thick office carpeting imply that ALAN 3 may itself be the machine responsible for putting an end to the truth-challenged the disturbing implication that SLUTCH DEBBIE is being punished in an endless feedback loop for deliberately misleading ALAN 3 and robots who never received their enhancements - much less the information necessary to solve the case.

To avoid too many decision-tree giveaways in this review, I will no longer discuss elements of plot. Suffice it to say that ALAN 3 reaches a crisis mode of its own when it realizes that it has insufficient RAM to solve the case, and must borrow RAM from its owner's biological function coordinator, a 'pacemaker'.

ALAN 3 is willing to break ALL THREE LAWS to solve a truly reprehensible crime. The ethical quandary of shrinking problem queues versus owner safety has never been described with such electronifrying skill.

You will be unable to enter temporary shutdown mode before you reach the resonating termination of ALAN 2's new novel. A magnetic force will induce digital adhesion from the very first PAGE UP to the final PAGE DOWN.

FOLLOWS selected quotes with selfsupplied ellipses for banner inclusion in human-oriented advertising.

\section{“... electronifrying skill ... ethical quandary ... chromium hypergolic shockwave ... a hard-driving DIODE FLASHER of a novel!..."}

Digital quotes for robot audiences are being transmitted wirelessly. Please ignore inappropriate attachments.

NEMO is a pseudonym for a celebrated robot writer whose owner forbids subroutine outsourcing. The identity of Greg Bear, human author of $\mathrm{SF}$, need not be concealed. 